\title{
Data-Oriented Process Development: Determination of Reaction Parameters by Small-Scale Calorimetry with in situ Spectroscopy
}

\author{
Graeme Puxty, Ulrich Fischer, Marc Jecklin, and Konrad Hungerbühler
}

\begin{abstract}
The rapid and complete characterization of chemical reaction mechanisms and their associated heat production is of the utmost importance in terms of chemical understanding and process safety and efficiency. In this contribution a new small volume $(25-50 \mathrm{ml})$ combined reaction calorimeter $(C R C)$ will be described. With this reactor it is possible to make calibration free calorimetry measurements and in situ mid-IR measurements simultaneously as well as gas consumption/production measurements in pressure-proof versions. The application of this reactor to the industrially relevant solvent free esterification of 1-butanol by acetic anhydride followed using calorimetry and mid-IR spectroscopy (a common reaction in the synthesis of many solvents, pharmaceuticals and perfumes) will be demonstrated. The results for this chemical system are presented in conjunction with a description of data analysis techniques allowing kinetic model fitting simultaneously to the different types of in situ measurements.
\end{abstract}

Keywords: Calorimetry $\cdot$ Kinetic model fitting $\cdot$ Process optimization · Spectroscopy

\section{Introduction}

The globalization of the chemical market, and as a result the growing competition, requires chemical producers to manufacture high-quality products at low costs. Additionally, the growing environmental concerns demand processes with the best possible conversion of energy and raw materials [1-4]. These criteria should be fulfilled by using short and efficient procedures in order to start production as soon as possible.

At the core of every chemical process there is an intended reaction generally accompanied by unwanted side reactions. The intended reaction may proceed in one single step or, more often, takes place in several chemical transformations. Also, side reactions may proceed in multiple steps thus leading to complex reaction schemes. Process development and process control aim at choosing operating conditions that favour

${ }^{\star}$ Correspondence: Prof. K. Hungerbühler ETH Zürich

Institute for Chemical and Bioengineering

$\mathrm{HCl}$ G133, Wolfgang-Pauli-Strasse 10

$\mathrm{CH}-8093$ Zürich

Tel.: +41446326098

Fax: +4144632 1189

E-Mail: hungerb@chem.ethz.ch

www.sust-chem.ethz.ch the synthesis of the main product and minimize unwanted by-products. A high yield signifies not only a higher economic profit from product sales but also an efficient use of raw materials, energy (e.g. less energy required for separations), and utilities, as well as the generation of less waste and lower emissions.

Process simulation is one efficient tool in process development. Chemical processes can be simulated and optimized on the basis of physical properties, kinetic and thermodynamic information, and unit operation models. Most common objective functions are designed to reach the maximum yield and selectivity for the desired product or to maximize the profit [5]. A fundamental understanding of the thermokinetics is also a prerequisite for an investigation of process safety [6].

Of course, most of the chemical reactions employed in the production of fine chemicals and pharmaceuticals are rather complex from a mechanistic point of view. However, it should be possible to propose reasonable empirical models for most of the reactions from basic chemical knowledge. An empirical reaction model has to fulfil the needs of the early process development, but does not have to represent deep insight into the actual reaction mechanism. Thus, an empirical reaction model only needs to describe the most important main and side reactions with as few reaction parameters as possible. This will minimize the effort needed to quantify the proposed parameters and increase the robustness of the model in the later application.

All reaction models will include initially unknown reaction parameters such as reaction orders, rate constants, activation energies, phase change rate constants, diffusion coefficients, and reaction enthalpies. Unfortunately, it is a fact that there is hardly any knowledge about these kinetic and thermodynamic parameters for a large majority of reactions in the production of fine chemicals and pharmaceuticals; this impedes the use of model-based optimization tools for individual reaction steps, so the identification of optimal and safe reaction conditions, for example, can be difficult.

Although many different analytical techniques have been developed during the past decades, and various mathematical algorithms exist to extract the desired information from experimental data, these methods nevertheless suffer from some fundamental drawbacks. For example, many analytical techniques requiring calibration and sampling still take too long; with regard to sampling, therefore, in situ analytical techniques offer an important advantage. Furthermore, not all of the desired reaction parameters can be measured directly and some can only 
be obtained by complex processing of the basic measurement data. Such determinations are often time consuming or require sophisticated mathematical techniques. In the early stages of process development, there might also be insufficient quantities of the essential test compounds available to carry out the required analyses. These facts call for a further development of the available analytical techniques, or the invention of new ones. Otherwise, considerable potential for the improvement of many chemical processes, which in fact needs to be achieved, might remain elusive.

In the following we will present a recently developed reactor and modelling approach based on small-scale reaction calorimetry and in situ mid-IR measurements for the determination of reaction parameters and will demonstrate the application of the device and modelling technique to the solvent-free esterification of 1-butanol by acetic anhydride.

\section{The Combined Reaction Calorimeter (CRC)}

Research in the area of reaction characterization and modelling can be broken down into three main categories. Research and development of devices for measurement, experimental studies of specific chemical systems, and data analysis tools for evaluating the data produced by measurements. The combined reaction calorimeter $(\mathrm{CRC})$ is a small volume $(25-50 \mathrm{ml})$ lab scale batch/semi-batch reaction calorimeter that has been under ongoing development for the last seven years [7-9]. It currently exists in three versions, each of which has design differences but shares the same principle of operation. Each CRC combines power compensation calorimetry and heat flow calorimetry [10]. This combination allows calibration-free time-resolved calorimetry measurements to be made as any changes in heat flow characteristics due to changes in volume or solution properties are measured directly and accounted for in the energy balance. The reactor also uses a copper jacket and its set-point temperature is controlled by Peltier elements. The use of a compensation heating system in conjunction with a thermoelectrically controlled metal jacket means a much faster response time and more consistent heat flow characteristics than traditional liquid jacket devices. The reactor combines calorimetry with in situ mid-IR spectroscopy via an ATR window. The two latest versions of the reactor are pressure-proof allowing the introduction of a gas handling system. This means for gas-liquid reactions such as hydrogenations, gas consumption can also be measured in situ. Additionally, the CRC allows probes of up to $6.35 \mathrm{~mm}$ (1/4 inch) diameter to be inserted via the lid for extra in-situ analytics (e.g. UV-visible spectroscopy or backscattering particle size measurements).

An overview of the CRC version 4 is shown in Fig. 1. The reactor is designed to run under isothermal operating conditions and has a removable reactor vessel constructed from Hastelloy®. The set-point temperature for the reactor vessel is controlled by a combination of the immersion heater, thermocouple and a PID control loop. The jacket temperature is controlled by a second independent PID control loop consisting of the Peltier elements and thermocouples placed around the jacket. The Peltier elements use the thermoelectric principle to pump heat from the side attached to the reactor jacket to the side attached to the heat exchanger. Heat is then removed from the Peltier elements outside the energy balance of the reactor via cooling water flowing through the heat exchangers. When operating in the power compensation mode the set-point temperature for the reactor vessel is set higher than the jacket temperature. This means at steady-state a constant amount of energy is being introduced via the heater to maintain the set-point temperature and there is a constant energy flow across the jacket to the Peltier elements. When a reaction is initiated, for example by pumping in of a reagent for a semi-batch reaction, the output of the heater is increased or decreased by the same amount of energy being consumed or evolved by the reaction. The amount of energy being removed from the jacket via the Peltier elements can also be determined from the known characteristics of the Peltier elements and the voltage and current applied to maintain the set-point temperature. The overall energy balance to yield the reaction power is given by Eqn. (1) where $q_{\text {react }}$ is the reaction power (W), $q_{\text {Peltier }}$ is the power being removed by the Peltier elements (W), $q_{\text {heater }}$ is the power output of the heater $(\mathrm{W}), q_{\text {dose }}$ is the power introduced via dosing of a reagent (can be calculated from the dosing temperature and heat capacity, W) and $q_{\text {loss }}$ is the power lost to the environment (can be calibrated independently of reactor content, W).

$$
q_{\text {react }}=q_{\text {Peltier }}-q_{\text {heater }}-q_{\text {dose }}+q_{\text {loss }}
$$

A graphical representation of all the terms from the energy balance is given in Fig. 2. Energy introduced via the stirrer has been excluded from this Eqn. as it was

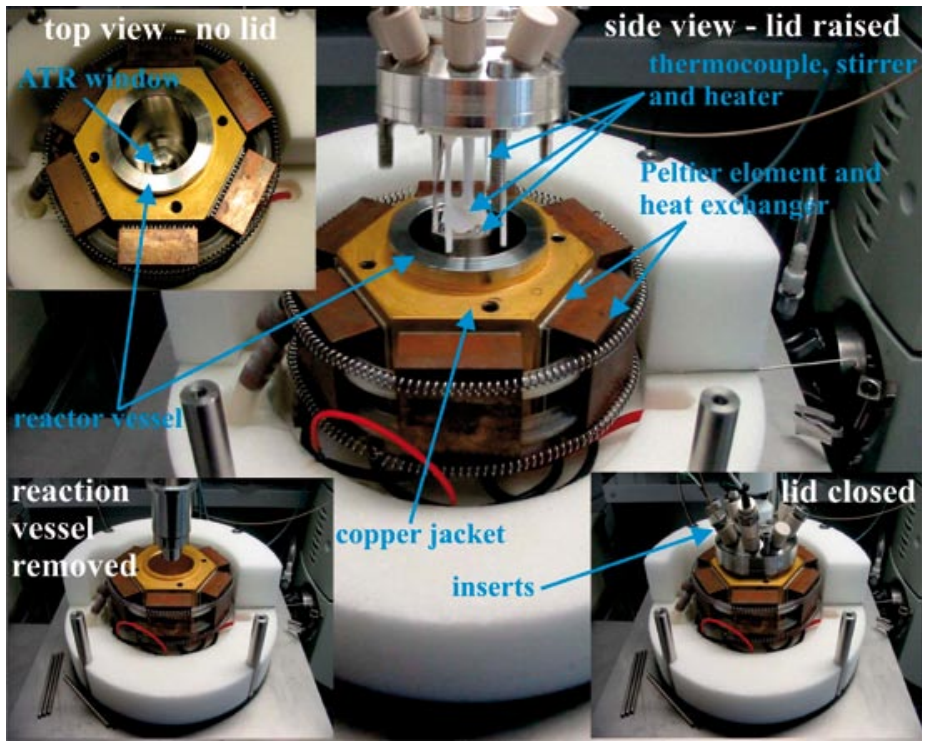

Fig. 1. Overview of the combined reaction calorimeter (CRC) version 4. The reaction vessel, thermocouple and heater are constructed from or coated

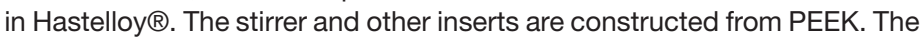
ATR window is a ZnSe crystal.

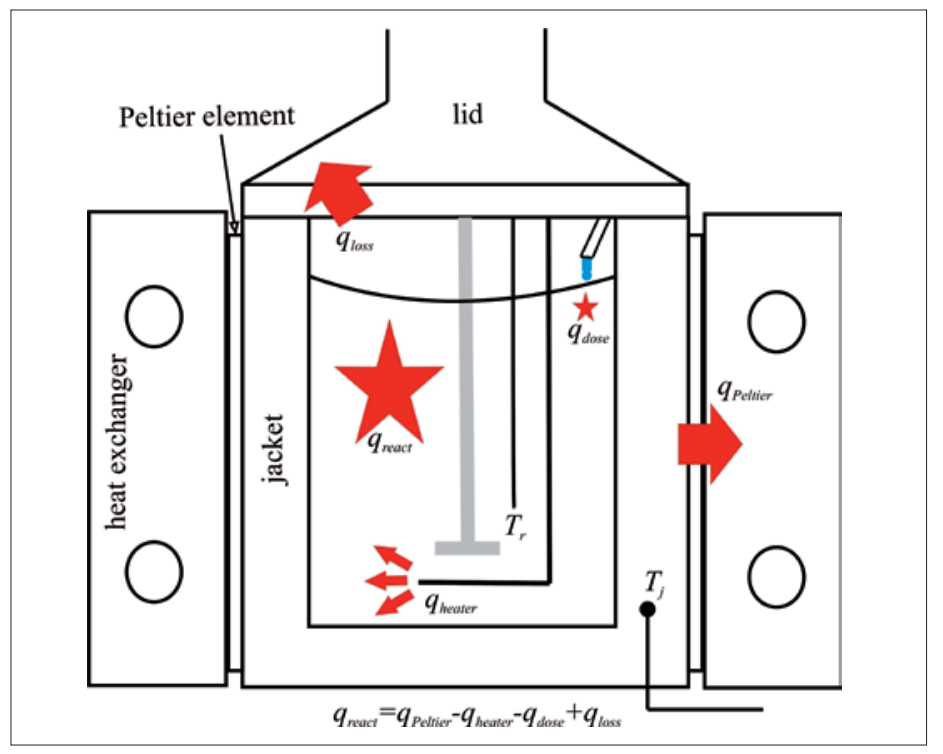

Fig. 2. Slice through the CRC version 4 showing the different components of the energy balance of Eqn. (1). Each of the energy terms is shown in red. The thermocouples for the measurement of the reaction temperature ( $\mathrm{Tr}$ ) and the jacket temperature (Tj) are also shown. 
determined to be negligible for the stirrer design used. For full details about the reactor the reader is directed to Visentin et al. [8]. Changes in volume or physical properties of the liquid inside the reactor vessel that lead to a change in the heat transfer coefficient are directly accounted for in the energy balance by a corresponding change in $q_{\text {Peltier }}$. The Peltier elements respond by removing more or less energy as required to maintain the jacket set-point.

The ZnSe ATR crystal integrated into the bottom of the reactor vessel allows in situ mid-IR spectra to be measured. In the setup shown in Fig.1 mid-IR spectra are measured using a ReactIR ${ }^{\mathrm{TM}} 4000$ system from Mettler-Toledo. The ability to measure mid-IR spectra in situ has a number of advantages and provides complimentary information to calorimetry, which is a bulk measurement of all thermal phenomena. Time-resolved spectroscopic data provides additional kinetic information and the appearance or disappearance of specific chemical species can often be observed. Furthermore, through the use of multivariate spectroscopic data (time-resolved spectra at multiple wavenumbers/wavelengths) a variety of factor analysis based self-modelling curve resolution techniques [11-15] and powerful techniques for the fitting of kinetic models [16][17] can be applied. The fitting of kinetic models to measured calorimetry and spectroscopic data will be demonstrated in the next section.

Besides the advantages associated with faster response time and improved heat flow characteristics the use of a metal reactor vessel and jacket allows the reactor to be easily pressure proofed and to operate at high pressures (up to 30 bar in version 4 and 70 bar in a new version under construction). The introduction of a gas handling system allows the gas consumption to be measured for gas-liquid reactions in conjunction with the other measurements. Also, for reactions evolving a gas, gas production can be measured via the pressure increase inside the reactor. The measurement of gas consumption for the hydrogenation of nitrobenzene and ethyl-4-benzoate has been demonstrated in Visentin et al. [18].

\section{Solvent-free Esterification of 1-Butanol by Acetic Anhydride}

The production of esters is an important synthetic step in many chemical processes and they are important precursors in the manufacture of many solvents, pharmaceuticals, perfumes and explosives [19]. Butyl acetate is produced from the esterification reaction between 1-butanol and acetic anhydride in the presence of an acid catalyst in many hundreds of thousands of tonnes per annum [20]. It is very important that such a large volume chemical is manufactured in the most environmentally efficient and safe manner possible. To this end manufacture without solvent is an attractive option as issues surrounding solvent disposal or recycling are eliminated. However, without solvent to act as a thermal buffer and mediate the reaction rate a good understanding of the reaction mechanism and associated reaction enthalpies are crucial for safe operation under solvent-free conditions. In this section the characterization of this reaction for a specific catalyst is shown. Characterization of the reaction mechanism in minimum time, reagent use and effort is shown through the use of factorial experimental design and the simultaneous fitting of a first principles chemical model to all spectroscopic and calorimetric measurements made using the $\mathrm{CRC}$.

\section{Experimental}

All experiments were carried out using 1-butanol $(\mathrm{BuOH},>98 \%$, Fluka) and acetic anhydride (AA, >99\%, Brunschwig Chemicals). The acid catalyst used was 1,1,3,3-tetramethylguanidine (TMG, >99 $\%$, Fluka). Prior to use all chemicals were dried for a minimum of $24 \mathrm{~h}$ using freshly prepared 4 A molecular sieves.

All experiments were carried out in the CRC version 3 using the following procedure. Details of the apparatus, which uses the same principle but has some design differences to that described above, can be found in Zogg et al. [9]. Following cleaning with acetone the reactor was sealed and

Table 1. Initial concentration following dosing of all reagents, volume dosed (in brackets) and reactor set-point temperature for all experiments in the factorial experimental design.

\begin{tabular}{|c|c|c|c|}
\hline$c_{\mathrm{BuOH}}: c_{\mathrm{AA}}[\mathrm{M}](\mathrm{ml})$ & Experiment \# & & $c_{\mathrm{TMG}}[\mathrm{M}](\mathrm{ml})$ \\
\hline 4.6:5.8 (18.6:24.0) & 1 & & $0.24(1.35)$ \\
\hline 5.3:5.3 (21.4:22.0) & 2 & & $0.12(0.65)$ \\
\hline 5.3:5.3 (21.4:22.0) & 3 & 5 & $0.22(1.25)$ \\
\hline $5.1: 5.1(20.4: 21.0)$ & 6 & & $0.43(2.35)$ \\
\hline $5.8: 4.7(21.4: 22.0)$ & 7 & & $0.19(1.05)$ \\
\hline Temperature $\left[{ }^{\circ} \mathbf{C}\right]$ & 30 & 50 & \\
\hline
\end{tabular}

purged with dry $\mathrm{N}_{2}$. The $\mathrm{CRC}$ was then charged with AA and TMG. The stirrer speed was set to $400 \mathrm{rpm}$ and the reactor was allowed to equilibrate to the set-point reaction temperature (the jacket set-point was $25{ }^{\circ} \mathrm{C}$ below the reaction set-point). The reaction was then initiated by dosing $\mathrm{BuOH}$ at a rate of $3 \mathrm{ml} / \mathrm{min}$. Data collection for analysis was begun six minutes prior to dosing of $\mathrm{BuOH}$ and the reaction was following by measurement of the mid-IR spectra (Bruker Equinox 55 FTIR and Axiom DMD 260 ATR probe) and the calorimetry signal.

As mentioned, factorial experimental design [21] was used to choose a range of experimental conditions that would allow satisfactory determination of the reaction rate constants, activation energies and enthalpies in a minimum of experiments. The experimental design is shown in Table 1.

\section{Results and Data Fitting}

Initially, the measurements were evaluated using the self-modelling curve resolution technique of alternating least squares with penalty functions [22]. Basically, it applies the alternating least squares algorithm to qualitatively resolve concentration profiles and pure components spectra from multivariate spectroscopic data. In addition this method applies user-defined constraints. The algorithm was applied to the spectroscopic data from each measurements over the wavenumber ranges 3709-2730 $\mathrm{cm}^{-1}$ and $1860-1200 \mathrm{~cm}^{-1}$. Other ranges were excluded from the analysis as they contained no usable data due to interference from the ATR crystal. The constraints used were non-negative and uni-modal concentration profiles. The results of applying this method to data from experiment 3 are shown in Fig. 3. The concentration profiles and pure component spectra are unit-less as they have not been scaled to any concentration data so the magnitude of each curve has no meaning. Three absorbing species capture $99 \%$ of the variance of the measured data. The concentration profiles suggest a simple mechanism where the reactants form a product(s) in a single step following dosing. It is not possible to distinguish between the simultaneous formation of multiple products as the concentration profiles are linearly dependent. The same concentration profile behaviour was observed for all measurements. There was no indication of the formation of any intermediate species and the reaction appeared to go to completion. The solid (-) and dashed (---) lines represent $\mathrm{AA}$ and $\mathrm{BuOH}$ respectively (the dosing of $\mathrm{BuOH}$ is clear) and the dotted (..) line represents the formation of butyl acetate (BuOA) and acetic acid ( $\mathrm{AH})$. This interpretation is supported by the pure com- 
ponent spectra. The spectrum given by the solid line shows the distinctive $\mathrm{C}=\mathrm{O}$ band for anhydrides at $1840-1740 \mathrm{~cm}^{-1}$ and no significant $\mathrm{C}-\mathrm{H}$ or $\mathrm{O}-\mathrm{H}$ stretching. Alternatively, the dashed spectrum shows the $\mathrm{C}$ $\mathrm{H}\left(3000-2800 \mathrm{~cm}^{-1}\right)$ and O-H (3600-3000 $\mathrm{cm}^{-1}$ ) stretching bands expected of 1-butanol. The dotted spectrum shows characteristics of both BuOA $(\mathrm{C}=\mathrm{O}$ band shifted to $\left.1740-1700 \mathrm{~cm}^{-1}\right)$ and $\mathrm{AH}(\mathrm{O}-\mathrm{H}$ stretch shifted to shorter wavenumbers). No contribution to the spectra from TMG is apparent as would be expected with the combination of the short in solution light path of an ATR probe and such a low concentration. This was confirmed by there being no change in the spectrum of AA (other than dilution) upon addition of TMG.

Using this result as a guide the simple reaction mechanism shown in Fig. 4 was postulated. The mechanism was fitted simultaneously to all the spectroscopic and calorimetric data using second-order global analysis (Table 2) [17][23]. The temperature dependence of the rate constant was taken into account using the Arrhenius model of Eqn. (2) where $k_{r e f}$ is the rate constant at the reference temperature $T_{r e f}(\mathrm{~K})$, $T$ is the temperature $(\mathrm{K}), E_{a}$ is the activation energy $(\mathrm{J} / \mathrm{mol})$ and $R$ is the universal gas constant $(8.314 \mathrm{~J} / \mathrm{mol})$.

$$
k=k_{r e f} e^{-\frac{E_{a}}{R}\left(\frac{1}{T}-\frac{1}{T_{r e f}}\right)}
$$

Use of the Arrhenius model in this form has been shown to yield reduced correla- tion between the pre-exponential factor and the activation energy [24] during optimisation. In summary, the ordinary differential equations (ODEs) required to describe the reaction kinetics and dosing are derived automatically from the model written in chemistry notation using a model parser. The resulting ODEs are shown in Eqn. (3) where $c_{X}$ is the concentration of species $\mathrm{X}$ (M), $f_{\text {in }}$ is the flow rate for the dosing of $\mathrm{BuOH}(1 / \mathrm{s}), c_{i n, \mathrm{BuOH}}$ is the concentration of $\mathrm{BuOH}$ in the flow and $V$ is the liquid volume (1) in the reactor.

$$
\begin{aligned}
& v=k \cdot c_{\mathrm{BuOH}} \cdot c_{\mathrm{AA}} \cdot c_{\mathrm{TMG}} \\
& \frac{d c_{\mathrm{BuOH}}}{d t}=-v+\frac{f_{i n}}{V} \cdot\left(c_{i n, \mathrm{BuOH}}-c_{\mathrm{BuOH}}\right) \\
& \frac{d c_{\mathrm{AA}}}{d t}=-v-\frac{f_{\text {in }}}{V} \cdot c_{\mathrm{AA}} \\
& \frac{d c_{\mathrm{BuOA}}}{d t}=v-\frac{f_{\text {in }}}{V} \cdot c_{\mathrm{BuOA}} \\
& \frac{d c_{\mathrm{AH}}}{d t}=v-\frac{f_{\text {in }}}{V} \cdot c_{\mathrm{AH}} \\
& \frac{d c_{\mathrm{TMG}}}{d t}=-\frac{f_{\text {in }}}{V} \cdot c_{\mathrm{TMG}} \\
& \frac{d V}{d t}=f_{\text {in }}
\end{aligned}
$$

Initially, estimates for the value of $k_{r e f}$ and $E_{a}$ are provided. Using these estimates and the known initial concentration of all
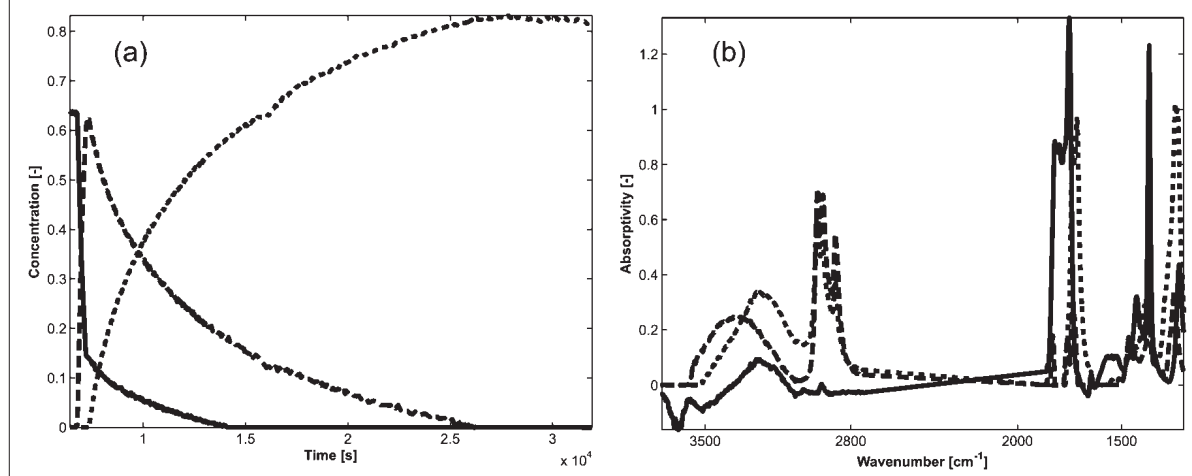

Fig. 3. The estimated concentration-time profiles (a) and pure component spectra (b) from the application of alternating least squares with penalty functions to the measured mid-IR spectra for experiment 3.

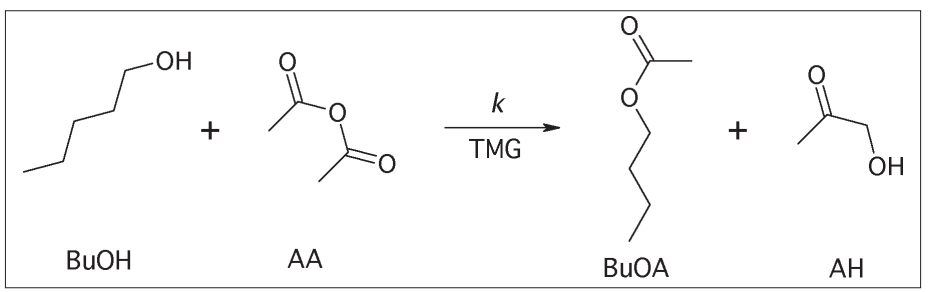

Fig. 4. Proposed simple mechanism to describe the solvent free esterification of 1-butanol. reactants, the ODEs are integrated to yield the time-resolved reaction rate, concentration profiles of all species and volume for all measurements. The concentration profiles are then fitted to the spectroscopic data by assuming Beer-Lambert's law and using linear least squares regression. The errors of fit for the spectroscopic data are shown in Eqn. (4) where $\mathbf{E}$ are the estimated pure component spectra of each absorbing species, $\mathbf{C}$ is the time resolved concentration profiles of absorbing species arranged into a matrix, $\mathbf{C}^{+}$ is the pseudo-inverse of $\mathbf{C}, \mathbf{A}$ is the spectroscopic data arranged into a matrix and $\mathbf{R}$ are the time and wavenumber dependent errors of fit arranged into a matrix.

$$
\begin{aligned}
& \mathbf{E}=\mathbf{C}^{+} \cdot \mathbf{A} \\
& \mathbf{R}=\mathbf{A}-\mathbf{C} \cdot \mathbf{E}
\end{aligned}
$$

The reaction rate is similarly fitted to the reaction power $q_{\text {react }}$. The errors of fit for the power data are given by Eqn. (5) where $\Delta \mathbf{h}$ are the reaction enthalpies $(\Delta H$, $\mathrm{J} / \mathrm{mol}$ ) of each step (in this case only one) arranged into a vector and $\mathbf{N}$ is the time-resolved reaction rate in $\mathrm{mol} / \mathrm{s}$ (reaction rate in $\mathrm{M} / \mathrm{s}$ multiplied by the volume) of each reaction step arranged into a matrix, $\mathbf{N}^{+}$is the pseudo-inverse of $\mathbf{N}, \mathbf{q}_{\text {react }}$ is the reaction power (W) arranged into a vector and $\mathbf{r}$ is the error of fit at each time point arranged into a vector.

$$
\begin{aligned}
& \Delta \mathbf{h}=\mathbf{N}^{+} \cdot \mathbf{q}_{\text {react }} \\
& \mathbf{r}=\mathbf{q}_{\text {react }}-\mathbf{N} \cdot \Delta \mathbf{h}
\end{aligned}
$$

The initial values of $k_{r e f}$ and $E_{a}$ were then iteratively optimized using the Newton-Gauss-Levenberg/Marquardt algorithm to minimize the sum of the squared error of $\mathbf{R}$ and $\mathbf{r}$ arranged into a single objective function. Extensive details of this method are given in Puxty et al. [17][23] and a similar method has been described by Zogg et al. [25]. Combining both objective functions into a single objective function is straightforward if both objective functions share essentially the same optimal parameter values, as was the case here. However, if there is some trade-off between the objective functions more complex multi-objective optimisation methods are required, such as that described in Gianoli et al. [26].

Table 2. Parameter values and their associated errors determined by fitting the simple mechanism of Fig. 4 to all experimental data simultaneously. See text for the determination of errors.

$\begin{array}{lll}\boldsymbol{k}_{\text {ref }} \text { at } 40{ }^{\circ} \mathrm{C}\left[\mathrm{M}^{-2} \mathrm{~s}^{-1}\right] & E_{\mathrm{a}}[\mathrm{kJ} / \mathrm{mol}] & \boldsymbol{\Delta} \boldsymbol{H}[\mathrm{kJ} / \mathrm{mol}] \\ 4.0 \pm 1 \times 10^{-4} & 37.3 \pm 0.01 & 43_{-8}^{+3}\end{array}$



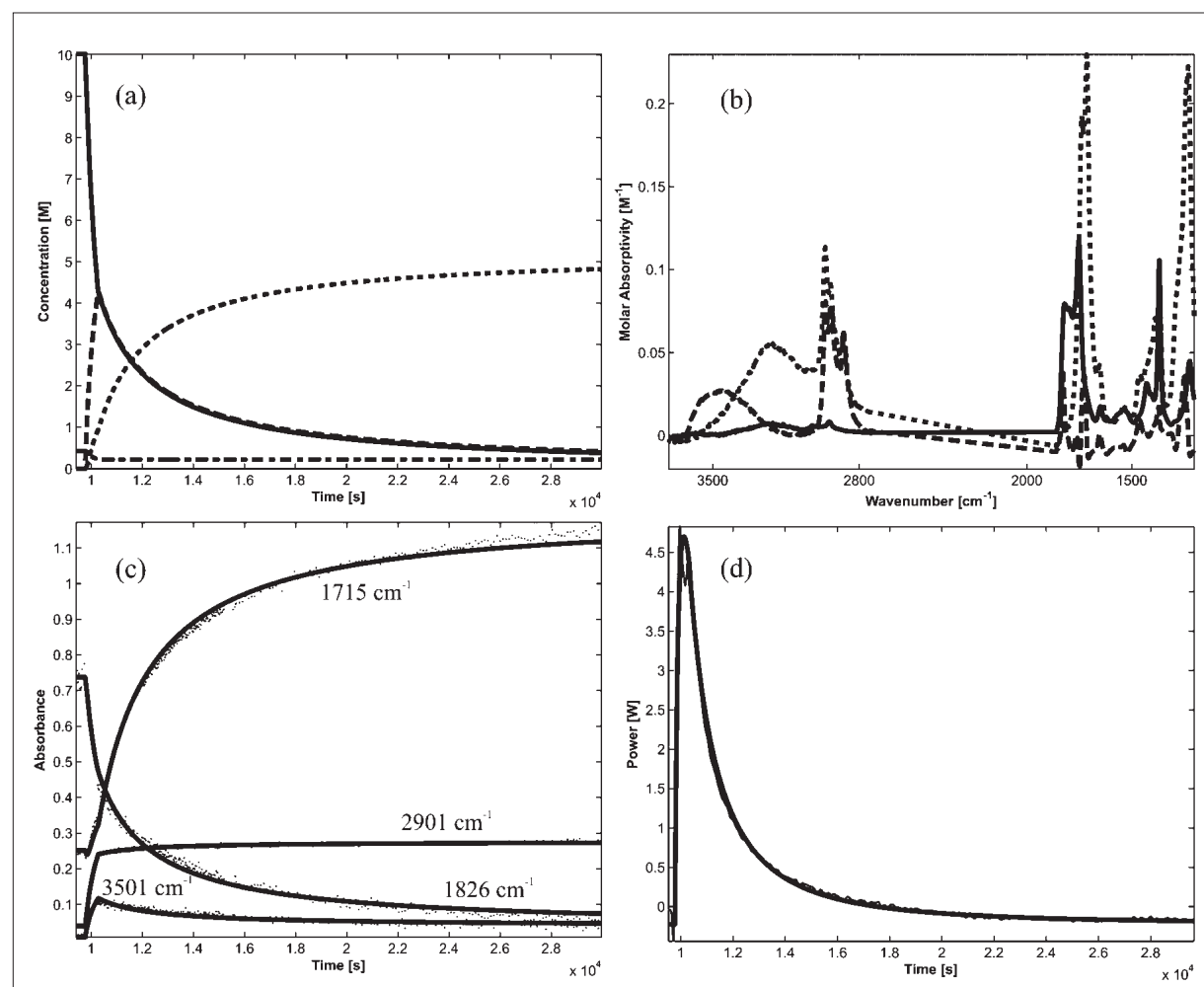

Fig. 5. Calculated data and fits for experiment 5 of Table 1: (a) and (b) are the calculated concentration profiles for AA $(-)$, BuOH (---), (BuOA+AH) (..) and TMG (--.--) and the calculated pure component spectra for $\mathrm{AA}, \mathrm{BuOH}$ and $(\mathrm{BuOA}+\mathrm{AH})$; (c) is the measured (grey, $\cdots$ ) and calculated $(-)$ absorbance data at selected wavenumbers; and $(d)$ is the measured (grey, $\cdots$ ) and calculated $(\rightarrow$ reaction power.

The use of concentration in molarity in Eqn. (3) does not take into account the changes in activity that could be expected when going from reactants to products under solvent-free conditions. However, the high quality of model fit obtained without taking activity changes into account indicates that attempting to do so would be fruitless in this case.

To account for the effect of temperature on the mid-IR spectra it was necessary to calculate a separate matrix of estimated pure component spectra for each experiment. This is known as the local spectra mode of analysis when using second-order global analysis [17]. This mode of analysis is necessary if there are measurement to measurement differences in the spectra. Three species were set as absorbing, $\mathrm{BuOH}$, $\mathrm{AA}$ and $(\mathrm{BuOA}+\mathrm{AH})$ as a single species, based on the initial self-modelling curve resolution results. The optimized parameter values are given in Table 2 . The error in $k_{r e f}$ was calculated as the range of $\mathrm{k}$ values from fitting the $40{ }^{\circ} \mathrm{C}$ experiments independently and for $E_{a}$ it is the statistical error of fit calculated from the Hessian matrix during nonlinear regression given to two standard deviations. The error in $\Delta H$ was calculated as the range from fitting each experiment independently.

The quality of fit obtained from the fitting of this model to all the experiments simultaneously is excellent with errors of fit approaching the instrumental noise level for both the spectroscopic and calorimetry data. The calculated concentration profiles, pure component spectra, fits at selected wavenumbers and fit of the power data for experiment 5 are shown in Fig. 5. The calculated concentration profiles and spectra are also in good agreement with the initial self-modelling curve resolution results. The fact that this model fits the spectroscopic and calorimetric data well for all seven experiments suggests that increasing the model complexity would yield no significant improvement in the quality of fit. To verify this, the model was extended to include an initial formation of either an $\mathrm{AA}-\mathrm{BuOH}$ or AA-TMG complex prior to the reaction. Any reaction between $\mathrm{BuOH}$ and $\mathrm{AH}$ to form $\mathrm{BuOA}$ and water was discounted as the spectra indicated no water was present. All more complex models yielded no significant improvement and could be immediately eliminated as a possibility. Further investigation of more complex mechanisms was considered unnecessary. Although this mechanism is certainly a simplification of a more complex series of steps, the fact that it fits all the measurements well means it represents what is the rate-determining step in the reaction.

The fact that this single and simple model can fit both the spectroscopic and calorimetric data for all seven experiments strongly supports that it will extrapolate ac- curately to conditions outside those of the seven experiments. To verify that this was the case two additional experiments were completed at $40{ }^{\circ} \mathrm{C}$ with half and double the minimum and maximum catalyst concentrations used respectively $\left(c_{\mathrm{TMG}}=0.058\right.$ and $0.81 \mathrm{M}$ ). Using the mechanism and parameter values determined with the seven experiments the concentration profiles and power profiles for these two measurements were simulated. The simulated concentration and power profiles were then fitted to the measured data according to Eqn. (4) and (5) without optimization. Excellent fits were obtained for both experiments confirming that the model showed good predictive power outside the range of the initial seven experiments. The calculated data and fits for the experiment with $0.81 \mathrm{M}$ of catalyst are shown in Fig. 6. The only significant deviation exists in the initial maximum amplitude of the power profile. This indicates there is an additional thermal phenomenon taking place not accounted for in the model, such as a change in the heat of mixing at the higher catalyst concentration. However, this small deviation aside, the ability of this model to predict the behaviour of the reaction outside the range of conditions used for the model determination highlights one of the main advantages of building up first principles kinetic models. This is of particular importance when using the model for batch process optimization.

\section{Conclusions}

The ability to determine a good reaction model for a chemical process is crucial for process design and optimization and to maintain safe operating conditions. As outlined in the introduction, many of the steps involved can only be carried out if a good reaction model and its associated parameters can be determined. Such a model developed from chemical understanding and using a minimum of complexity can provide excellent prediction of the reaction behaviour, even outside the range of conditions used for the model development.

The application of a new combined reaction calorimeter capable of measuring calorimetry and mid-IR spectroscopy to the characterization of the solvent-free esterification of 1-butanol by acetic anhydride has been shown. Initially, a minimum run of experiments, planned using factorial design, was made using the CRC. The spectroscopic data was then analysed using self-modelling curve resolution. This allowed postulation of a simple chemical model. The postulated model was then directly fitted to the measured spectroscopic and calorimetric data and its parameters were determined and an excellent fit to all the measured data was obtained using the model. To verify 

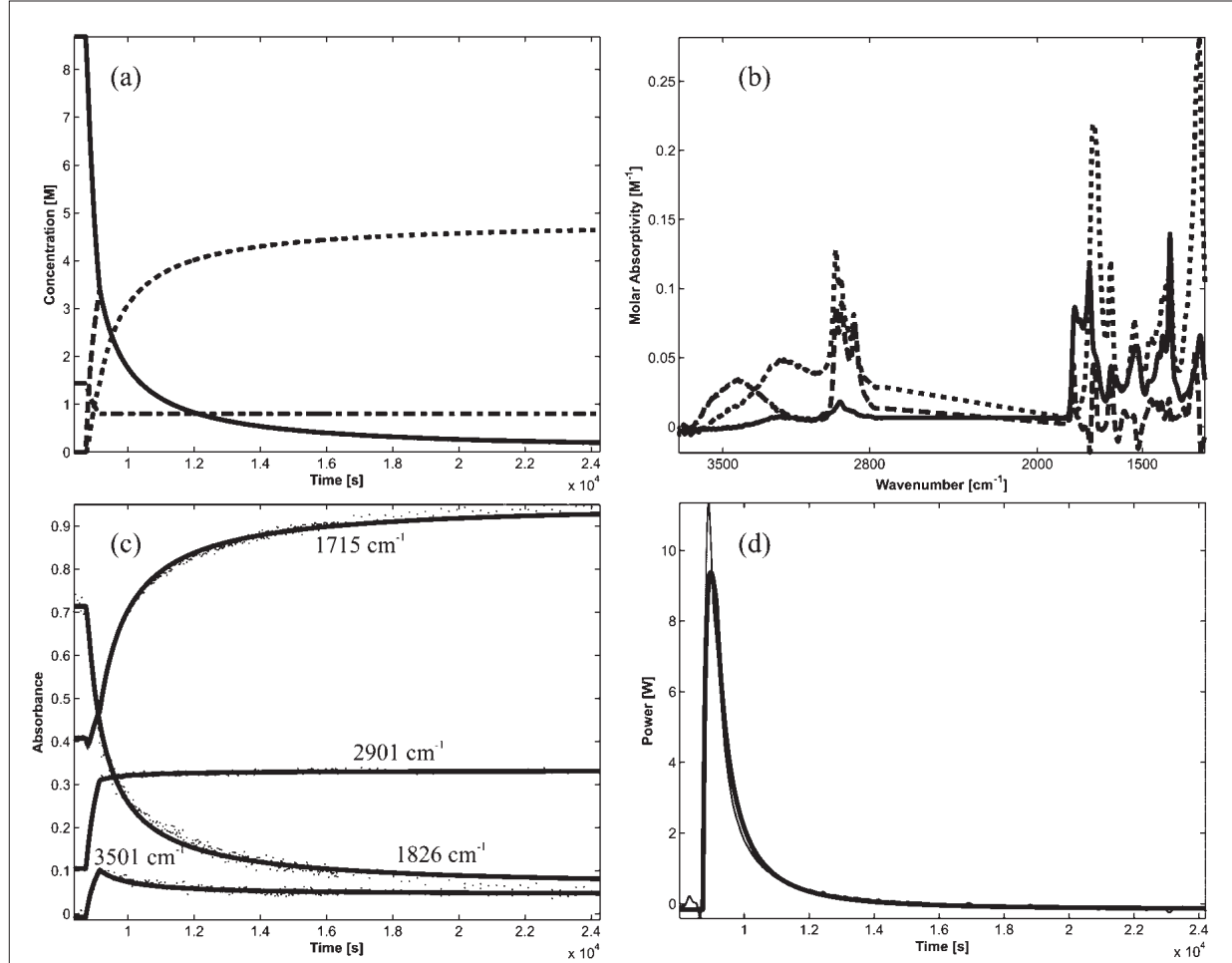

Fig. 6. Calculated data and fits for additional experiment at $40^{\circ} \mathrm{C}$ with $c_{\mathrm{BuOH}}=4.8 \mathrm{M}, \mathrm{c}_{\mathrm{AA}}=4.9 \mathrm{M}$ and $c_{\mathrm{TMG}}=0.81 \mathrm{M}$ using parameters of Table 2

the usefulness of the model, two additional experiments were made with catalyst concentrations outside the range of the initial experiments. The model was able to predict very accurately the behaviour of these two experiments.

It has been shown that with the combination of careful experimental design, a small-volume reactor with multiple in situ analytics and modern data analysis techniques chemical models that can predict reaction behaviour outside of the conditions used for model determination and suitable for use in process design and optimization can be rapidly developed. This approach eliminates many of the existing bottlenecks in the process development phase and minimizes reagent usage while extracting maximum information. Such an approach can be further developed by using additional in situ analytics and the further development of data analysis techniques that are capable of handling a variety of analytical signals in a consistent and user-friendly way.

Received: June 16, 2006

[1] U. Fischer, K. Hungerbühler, Chimia 2000, 54, 494-500.

[2] E. Heinzle, K. Hungerbühler, Comput. Chem. Eng. 1997, 51, 176-183.

[3] E. Heinzle, D. Weirich, F. Brogli, V.H. Hoffmann, G. Koller, M.A. Verduyn, K. Hungerbühler, Ind. Eng. Chem. Res. 1998, 37, 3395-3407.
[4] G. Koller, U. Fischer, K. Hungerbühler, Ind. Eng. Chem. Res. 2000, 39, 960-972.

[5] C. Guntern, A.H. Keller, K. Hungerbühler, Ind. Eng. Chem. Res. 1998, 37, 40174022.

[6] A.H. Keller, D. Stark, H. Fierz, E. Heinzle, K. Hungerbühler, J. Loss Prev. Process Ind. 1997, 10, 31-41.

[7] J. Pastre, A. Zogg, U. Fischer, K. Hungerbuhler, Org. Process Res. Dev. 2001, 5, 158-166.

[8] F. Visentin, S.I. Gianoli, A. Zogg, O.M Kut, K. Hungerbuhler, Org. Process Res. Dev. 2004, 8, 725-737.

[9] A. Zogg, U. Fischer, K. Hungerbuhler, Ind. Eng. Chem. Res. 2003, 42, 767-776.

[10] A. Zogg, F. Stoessel, U. Fischer, K. Hungerbuhler, Thermochim. Acta 2004, 419, $1-17$.

[11] M. Maeder, Anal. Chem. 1987, 59, 527530.

[12] E.R. Malinowski, 'Factor Analysis in Chemistry', 2nd ed.; John Wiley \& Sons, Inc., New York, 1991.

[13] E.R. Malinowski, J. Chemom. 1996, 10, 273-279.

[14] C.J. Mason, M. Maeder, A. Whitson, Anal. Chem. 2001, 73, 1587.

[15] R. Tauler, Chemom. Intell. Lab Sys. 1995, 30, 133-146.

[16] M. Maeder, Y.-M. Neuhold, G. Puxty, P. Gemperline, Chemom. Intell. Lab Sys. 2006, in print.

[17] G. Puxty, M. Maeder, K. Hungerbühler, Chemom. Intell. Lab Sys. 2006, 81, 149-164.
[18] F. Visentin, G. Puxty, O.M. Kut, K. Hungerbühler, Ind. Eng. Chem. Res. 2006, accepted.

[19] J.G. Speight, 'Chemical and Process Design Handbook', McGraw-Hill, New York, 2002.

[20] OECD Integrated HPV Database, OECD, 2006, http://cs3-hq.oecd.org/scripts/hpv/.

[21] T. Lundstedt, E. Seifert, L. Abramo, B. Thelin, A. Nystrom, J. Pettersen, R. Bergman, Chemom. Intell. Lab Sys. 1998, 42, 3-40.

[22] P.J. Gemperline, E. Cash, Anal. Chem. 2003, 75, 4236-4243.

[23] G. Puxty, M. Maeder, R.R. Rhinehart, S. Alam, S. Moore, P.J. Gemperline, J. Chemom. 2005, 19, 329-340.

[24] E. Furusjö, O. Svenssen, L.-G. Danielsson, Chemom. Intell. Lab Sys. 2003, 66 , $1-14$.

[25] A. Zogg, U. Fischer, K. Hungerbuhler, Chemom. Intell. Lab Sys. 2004, 71, 165 176.

[26] S. Gianoli, G. Puxty, U. Fisher, M. Maeder, K. Hungerbühler, Chemom. Intell. Lab Sys. 2006, in press. 